\title{
The Accuracy of Ground-Based Optical Interferometry Observations
}

\author{
Donald J. Hutter ${ }^{1}$ \\ Astrometry Department, U.S. Naval Observatory, Washington, DC \\ 20392
}

\begin{abstract}
The problem of wide-angle astrometry via interferometry is to recover the two coordinates for each star from the observed delays. In the absence of atmospheric turbulence, the geometrical delay can be defined as: $d_{G, i j}(t) \equiv d_{j}(t)-d_{i}(t)=\mathbf{B}_{i j}(t) \cdot \hat{\mathbf{s}}_{0}-C_{i j}$. Here the geometrical delay is the difference between the delay line lengths $d_{i}$ and $d_{j}$ that is required to equalize the effective optical paths from the star to the point of beam combination via each of two apertures $i$ and $j . \mathbf{B}_{i j}(t)$ is the baseline between the apertures, $\hat{\mathbf{s}}_{0}$ is the star position, and $C_{i j}$ is the difference between the 'fixed' internal optical path lengths $C_{i}$ and $C_{j}$ within the instrument. In principle, sufficient delay measurements would allow solution for the baseline vectors and the delay constants, as well as the positions of the stars. However, the actual situation is greatly complicated by the presence of the atmosphere and the fact that neither the delay 'constants' $C_{i j}$ nor the baseline vectors are stable over time. The design of, and the analysis of the data from, any ground-based optical interferometer must overcome all three of these effects. The design, operation, and the analysis of data from the Navy Prototype Optical Interferometer (NPOI) are presented here as examples of how to overcome the effects of the atmosphere and the instrumental instabilities in order to achieve accurate wide-angle astrometry. The status of the implementation of these techniques at the NPOI is presented.
\end{abstract}

\section{Introduction}

The Navy Prototype Optical Interferometer (NPOI; Armstrong et al., 1998a), located on Anderson Mesa, AZ (Figure 1), is a joint project of the U.S. Naval Observatory and the Naval Research Laboratory, in cooperation with the Lowell Observatory. The NPOI includes arrays for imaging and for astrometry. The imaging array consists of six movable $50 \mathrm{~cm}$ siderostats feeding $12 \mathrm{~cm}$ apertures, with baseline lengths from $2.0 \mathrm{~m}$ to $437 \mathrm{~m}$. The astrometric array consists of four fixed $50 \mathrm{~cm}$ siderostats feeding $12 \mathrm{~cm}$ apertures (soon to be increased to 35 $\mathrm{cm}$ ), with baseline lengths from $19 \mathrm{~m}$ to $38 \mathrm{~m}$. The arrays share vacuum feed and delay line systems. The NPOI features rapid tip-tilt star tracking, active groupdelay fringe tracking over a wide band (450-850 nm in 32 channels; Benson et

${ }^{1}$ Mailing address: P.O. Box 1149, Flagstaff, AZ 86002-1149; E-mail: djh@sextans.lowell.edu 


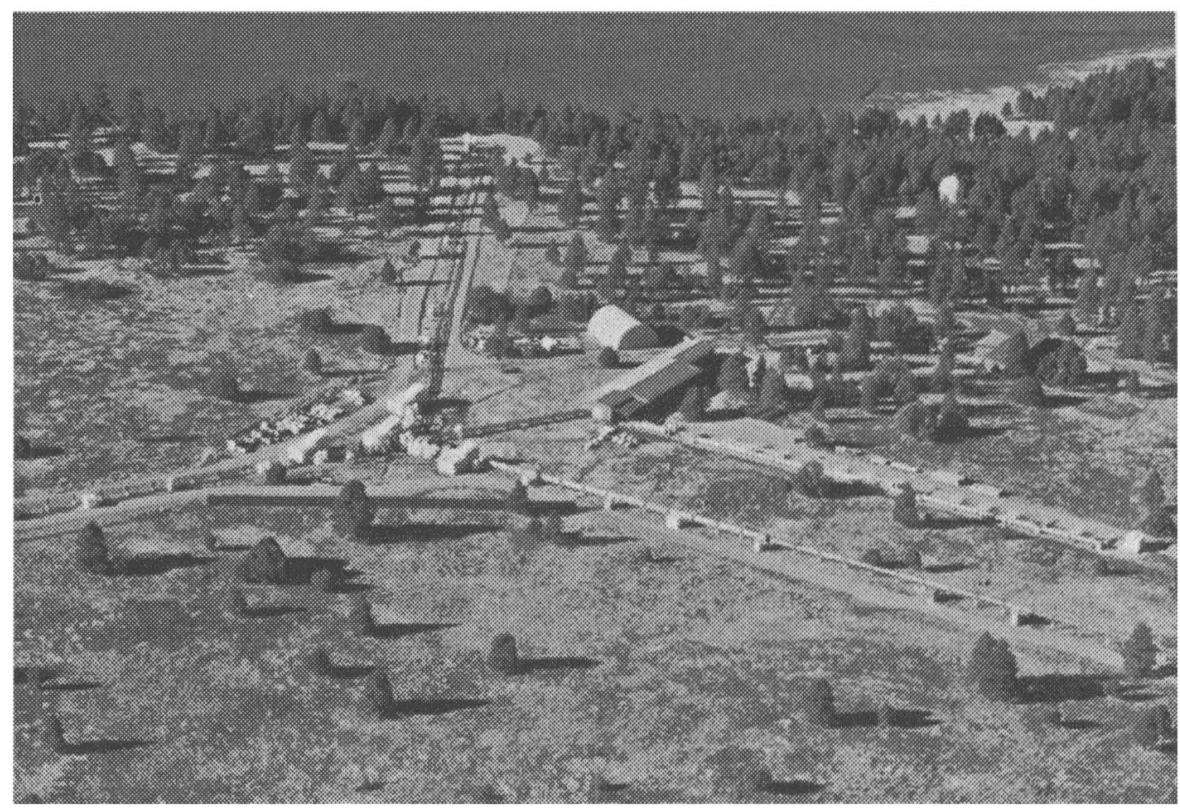

Figure 1. An aerial view of the NPOI showing the array as viewed from the northeast. The road surrounding the array can be seen along with parts of the north (right) west (center) and east (left) arms of the imaging array (station piers and vacuum feed lines visible). The astrometric siderostat shelters are the white structures at the array center. The beam-combining laboratory is the long structure to the right of the array center, with the 'long' delay line vacuum tanks (under construction) extending towards the north. The interferometer control building appears at the far right-center.

al., 1998), and a high degree of automation. The astrometric array includes an extensive baseline metrology system to measure the motions of the siderostats with respect to the local bedrock to $100 \mathrm{~nm}$ accuracy. Additional details of the NPOI design can be found in Armstrong et al. (1998b), Clark III et al. (1998), Mozurkewich (1994), and White et al. (1998).

The initial goal of wide-angle astrometric observations with the NPOI will be to produce a catalog of positions for $\sim 1000$ of the brighter HIPPARCOS stars with an internal accuracy of 1-3 mas. Astrometric observations of radio stars will be used to orient the NPOI catalog with respect to the fundamental reference frame defined by extragalactic radio sources. With an anticipated operational lifetime of more than a decade, the NPOI will significantly improve the measured proper motions of these (and additional) stars. (HIPPARCOS positional accuracies will have already degraded to $\sim 10$ mas by 2001 due to proper motion uncertainties.) Position measurements repeated at regular intervals will also allow unambiguous separation of binary motion from proper motion, an ac- 
complishment that might be difficult to achieve from space-based observations that are likely to be repeated only at intervals of decades. The NPOI catalog will also be used to check for and correct any systematic rotation in the HIPPARCOS reference system (estimated at up to $0.25 \mathrm{mas} / \mathrm{yr}$ ), and will be used to check for, and possibly correct, proper-motion induced zonal systematics in the HIPPARCOS system.

Thus, the NPOI, with a capability of milliarcsecond astrometry and a long, continuous operational lifetime, will be capable of maintaining the optical reference frame by improving the proper motions of thousands of the brighter HIPPARCOS stars through repeated observations.

\section{Wide-Angle Astrometry}

The problem of astrometry via interferometry is to recover the two coordinates for each star from the observed delays. In the absence of atmospheric effects, the geometrical delay can be defined as:

$$
d_{G, i j}(t) \equiv d_{j}(t)-d_{i}(t)=\mathbf{B}_{i j}(t) \cdot \hat{\mathbf{s}}_{0}-C_{i j} .
$$

The geometrical delay is the difference between the delay line lengths $d_{i}$ and $d_{j}$ that is required to equalize the effective optical paths from the star to the point of beam combination via each of two apertures $i$ and $j$. The vector $\mathbf{B}_{i j}(t)$ is the baseline between the apertures, $\hat{\mathbf{s}}_{0}$ is the star position unit vector, and $C_{i j}$ is the difference between the 'fixed' internal optical path lengths $C_{i}$ and $C_{j}$ within the instrument (which are independent of wavenumber in the case of the NPOI since the optical paths are in vacuum). In principle, sufficient delay measurements would allow solution for the baseline vectors and the delay constants, as well as the positions of the stars (e.g., Hummel et al., 1994). However, the actual situation is greatly complicated by the presence of the atmosphere, and the fact that neither the delay 'constants' $\left(C_{i j}\right)$, nor the baseline vectors are stable over time. Thermal drifts in the positions of siderostats, and all subsequent elements in the optical paths prior to the point of beam combination, typically produce drifts in the delay 'constants' of up to tens of $\mu \mathrm{m} /$ hour. Mechanical imperfections in the siderostats can produce $\sim 10 \mu \mathrm{m}$ changes in the baseline vectors even between successive observations of widely spaced stars! Finally, the atmosphere also induces large $(\geq \mu \mathrm{m})$, delay fluctuations on timescales as short as milliseconds. The design of the instrument and data analysis for the NPOI attempts to overcome all three of these effects.

\section{Baseline Metrology}

The design goal of the astrometric array of the NPOI is to measure stellar positions to 1-3 mas precision over the entire sky accessible from Anderson Mesa. This precision depends on knowing the 19-38 $\mathrm{m}$ astrometric baselines to $\sim 100 \mathrm{~nm}$. Since the baselines are not stable to that degree, we must measure changes due to such effects as thermal drifts and mechanical imprecisions in the siderostat mounts and correct for them in the astrometric solution (Eq. 1). The 
baseline metrology system (Hutter 1992, Elias 1994), described in Figure 2, is designed to monitor the motions of the siderostats with respect to local bedrock and to one another, to the required level of precision. This system consists of a number of laser interferometers tied to four temperature-stabilized super-Invar reference plates, one next to each siderostat. Five laser interferometers measure the position of a "cat's-eye" retroreflector (Danchi et al., 1986), near the intersection of the rotation axes of each siderostat, relative to the adjacent reference plate $^{1}$. Typical results for the observed motion of the siderostat retroreflector are shown in Figure 3. The rotation and tilt of the reference plates are, in turn, measured by two other metrology subsystems. In the first case, six laser interferometers monitor changes in the distances of three points on each plate from retroreflectors embedded in a deep subsurface layer. The second subsystem consists of interferometers along lines of sight between the reference plates that detect motions of the plates in the horizontal plane. Most of the length of each laser interferometer line of sight is in vacuum. Corrections are applied to the laser metrology data for changes in the optical path length due to variations of the temperature of the transmissive optics (and the variations in temperature, pressure, and relative humidity of the small remaining air paths).

Together, these various subsystems contain 56 laser interferometers that will allow a continuous measurement of the time evolution of the baselines with respect to an Earth-fixed reference system with sub-micron error.

\section{Constant Term}

Several techniques can be used to measure the temporal variations in the delay zero-point offset ('constant term') on each interferometer baseline. If rapid and/or discontinuous path length variations are present, then an internal laser metrology system must be used to continuously monitor the optical paths through the instrument. A single color (preferably infrared) metrology system could be employed to measure the relative changes in the paths, in combination with occasional fringe tracking observations of an internal white-light source, with the siderostats in autocollimation. Alternatively, a two-color, absolute metrology system could be employed. Both these systems pose significant technical challenges, including, for example, providing a retroreflector near the siderostat pivot that can be used simultaneously for internal path monitoring and baseline metrology. Fortunately, in the case of the NPOI, the temporal variations in the 'constant terms' are relatively slow and continuous.

In this case, it is practical to determine the differential optical path variations in the vacuum feed system and beam combining optics by periodic $(\sim 30$ min) white-light observations, with the siderostats in autocollimation, and determine those due to siderostat motion from measurements from the baseline

\footnotetext{
${ }^{1}$ Since it can be shown (Hines et al., 1990) that any point on the siderostat mirror surface can, if consistently used, be defined as one end of the baseline vector, the motion of a retroreflector perpendicular to the mirror surface can be monitored to determine the temporal variations of the baseline vector. In practice, the retroreflector is placed as close to the siderostat pivot as possible (within a few $\mu \mathrm{m}$ ) to allow monitoring of its position with laser beams of fixed orientation.
} 


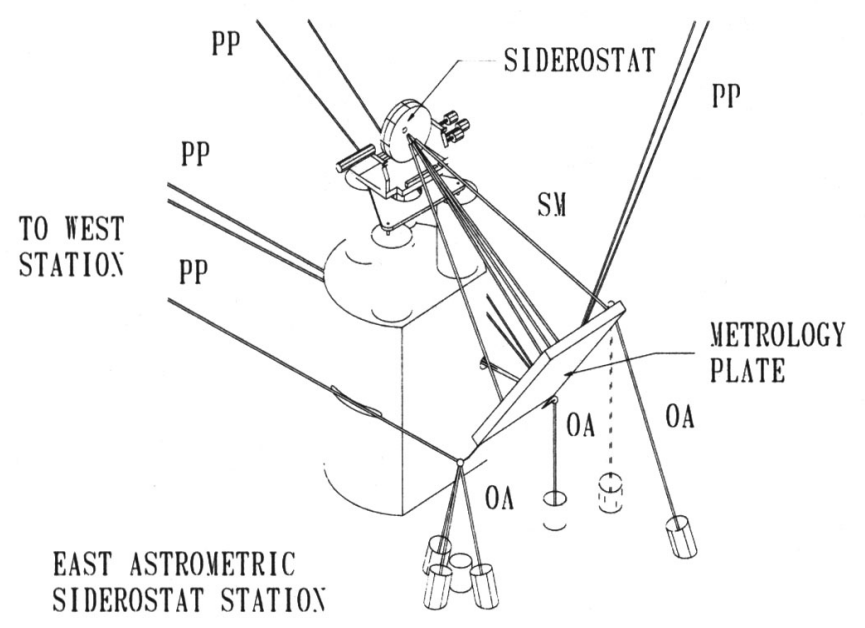

Figure 2. The baseline metrology system of the NPOI consists of 56 laser interferometers configured (in three subsystems) to monitor changes in the locations of the siderostats, in three dimensions, with respect to the local bedrock. This elevation view of the east astrometric siderostat station shows parts of the three metrology subsystems: the siderostat metrology (SM; five beams), which monitors the distance from the metrology plate to the siderostat pivot point; the optical anchor metrology (OA; seven beams), which monitors the motion of the metrology plate with respect to bedrock $7 \mathrm{~m}$ below the surface; and the pier-to-pier metrology (PP; seven beams), which monitors the horizontal motions of the metrology plates with respect to one another. 


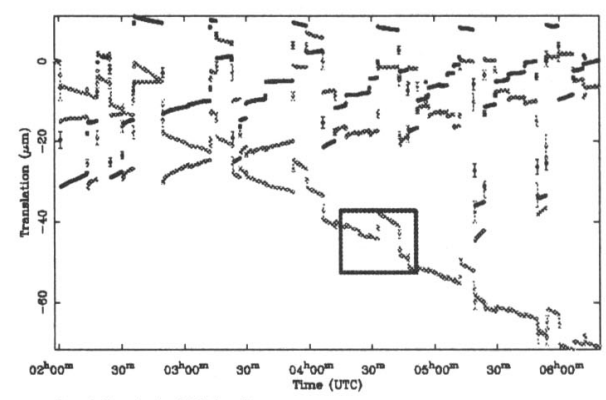

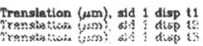
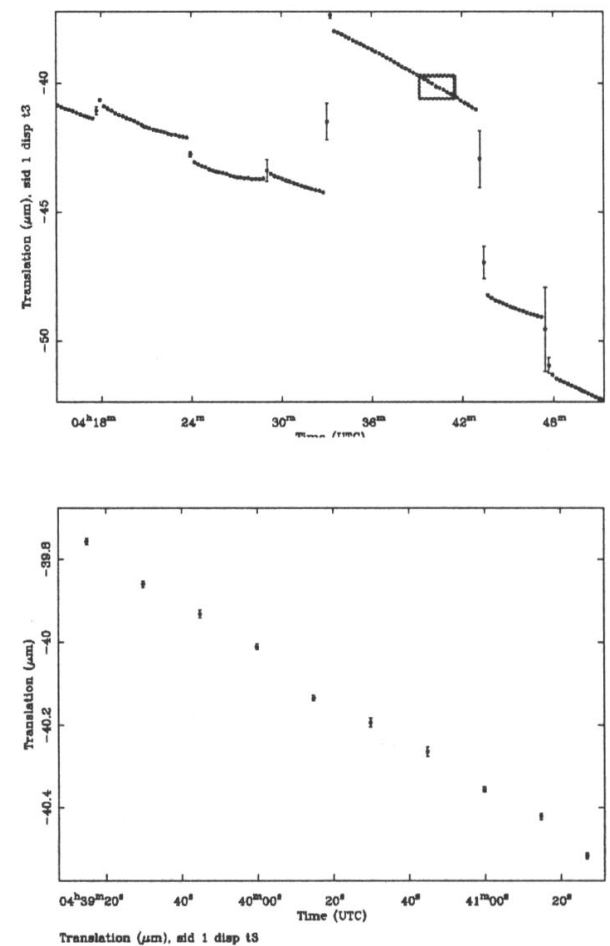

Figure 3. The motion of one siderostat pivot vs. time, derived from siderostat metrology. The points plotted in Figure 3a (top) represent the components of the pivot-point motion in the east, north, and vertical directions (upper, middle, and lower traces, respectively). The data show both the characteristic long term drifts due to thermal contraction of the siderostat and its pier over the course of the night, and the shorter-term step-like changes (observed when the siderostat slews between stars) due to mechanical imperfections of the siderostat that affect the location of the siderostat pivot. Figures $3 \mathrm{~b}-\mathrm{c}$ (middle and lower panels, respectively) show successive enlargements of a section of one of the plots to illustrate the typically small formal errors of the derived siderostat motion (typically $5-10 \mathrm{~nm}$ ). 
metrology system ${ }^{2}$ : The data from the white-light observations can be interpolated to the time of each stellar observation, then corrected for the siderostat pivot position at the time of that observation.

\section{Compensation of Atmospheric Effects}

The correction of the delay variations caused by atmospheric turbulence relies on the fact that, in the optical, atmospheric dispersion varies in a significantly nonlinear manner with wavelength. The method outlined below is a generalization of the "two-color" method (e.g., Colavita et al., 1987) that was successfully applied to astrometric data from the Mark III interferometer (Hummel et al., 1994).

The path change in the atmosphere above an array element $i$ is equivalent, to good approximation, to replacing a length $P_{i}$ of the vacuum path with air. The change in equivalent path length is given by $A_{i}(\sigma, t)=P_{i}(t)[n(\sigma)-1]$, where $n(\sigma)$ is the refractive index of air and $\sigma$ is the wavenumber. The observed delay - the delay required for effective path-length equality - is then given by:

$$
d_{i j}(\sigma, t)=\mathbf{B}_{i j}(t) \cdot \hat{\mathbf{s}}_{0}-C_{i j}-A_{i j}(\sigma, t)=d_{G, i j}(t)+d_{A, i j}(\sigma, t),
$$

where $A_{i j} \equiv A_{j}-A_{i}$, and the atmospheric delay $d_{A, i j}$ is the delay required to compensate for $A_{i j}$.

The observed phase $\phi_{i j}$, measured with respect to the direction $\hat{\mathbf{s}}_{0}$ is then given by:

$$
\phi_{i j}(\sigma, t)=\phi_{S, i j}(\sigma)+\phi_{A, i j}(\sigma, t),
$$

where the atmospheric phase $\phi_{A, i j}$ is defined by:

$$
\phi_{A, i j}(\sigma, t) \equiv 2 \pi \sigma d_{A, i j}(\sigma, t)=-2 \pi \sigma\left[A_{j}(\sigma, t)-A_{i}(\sigma, t)\right] .
$$

The variation of $\phi_{A, i j}(\sigma, t)$ with $\sigma$ can then be used to estimate $A_{i j}$, because $n(\sigma)-1$ has a significant $\sigma^{2}$ dependence at optical wavelengths. If we expand $\phi_{A, i j}$ as a Taylor series around some $\sigma_{0}$, we obtain:

$$
\phi_{A}(\sigma)=\phi_{A}\left(\sigma_{0}\right)\left[D_{1}+\sigma D_{2}+\Phi(\sigma)\right]
$$

where the $D$ coefficients depend on $\sigma_{0}, n\left(\sigma_{0}\right)-1, d n / d \sigma$, and $d^{2} n / d \sigma^{2}$. The first term gives a constant offset in phase. The second term produces a phase term that is indistinguishable from an error in the position of the star. The $\Phi(\sigma)$ term makes it possible to determine the air path mismatch. With the air path

\footnotetext{
${ }^{2}$ The component of the motion of the siderostat pivot in the direction of the siderostat feed contributes to the variations in the 'constant' terms.
} 
mismatch determined, we can calculate $\phi_{A, i j}(\sigma)$ and subtract it from $d_{i j}(\sigma)$, resulting in an estimate of $d_{G, i j}$, which is the desired datum for determining the stellar positions (Eq. 1).

The NPOI, with 32 spectral channels, is well-adapted for the application of this technique for the correction of atmospheric effects. The use of vacuum delay lines renders the interferometer insensitive to plane-parallel atmospheric refraction, and allows simultaneous fringe tracking in spectral channels over the entire optical bandpass. Fringe tracking on the NPOI is implemented via a group-delay tracking technique, which makes use of the fringes across a spectrum of the combined light, along with delay modulation. The delay on each baseline is modulated by a small number of wavelengths while, for each spectral channel and baseline, the photon count rates in eight bins per wavelength of modulation are synchronously measured. (Data are collected in this way for $2 \mathrm{~ms}$, a period short compared to the temporal coherence time of the atmosphere.) The complex Fourier transform of each set of bin counts provides the complex fringe visibility for that channel. A second Fourier transform over all the channels yields the group delay used for fringe tracking. Knowledge of the group delay for each 2 ms period allows one to rotate the complex visibility phasors by $e^{(2 \pi i d \sigma)}$, where $d$ is the group delay and $\sigma$ is the wavenumber of the channel. This allows the coherent addition (averaging) of the data to provide sufficient signal-to-noise to determine the variation of the fringe phase with wavenumber and thus apply the dispersion correction. All the data reported here were coherently averaged to $200 \mathrm{~ms}$. Application of the dispersion corrections typically results in a fourto eight-fold reduction in the RMS variations of the delays within an individual scan for bright stars. Results of dispersion correction on typical stellar data are shown in Figure 4.

\section{Current Status}

The implementation of baseline metrology, constant-term measurement, and correction of atmospherically induced delay fluctuations has advance to the point that accurate wide-angle observations with the NPOI are near-at-hand.

Dispersion compensation of the internal optical path observations of the white-light source, which are affected by delay fluctuations due to the air paths between the siderostats and the vacuum feed system (and to a much lesser extent the air paths in the vicinity of the beam combiner), can now be corrected to $\sim 300 \mathrm{~nm}$ RMS. This correction results in white-light fringe tracking data that vary sufficiently smoothly to allow accurate interpolation of the instrumental delay offset to the time of the stellar observations from white-light observations spaced at practical intervals $(\sim 30 \mathrm{~min})$.

Dispersion compensation of stellar observations, resulting in a reduction in the RMS fluctuations of observed delays to $\sim 500 \mathrm{~nm}$ per (typically 90 second) observation, has been achieved only for bright (third magnitude) stars. However, improvements in the techniques for coherently averaging the $2 \mathrm{~ms}$ fringe data promise similar accuracies in the atmospheric compensation of observations of much fainter stars in the near future.

To date, measurements of siderostat pivot motion have demonstrated repeatability at the $\sim 200 \mathrm{~nm}$ level, while known, externally-applied baseline mo- 

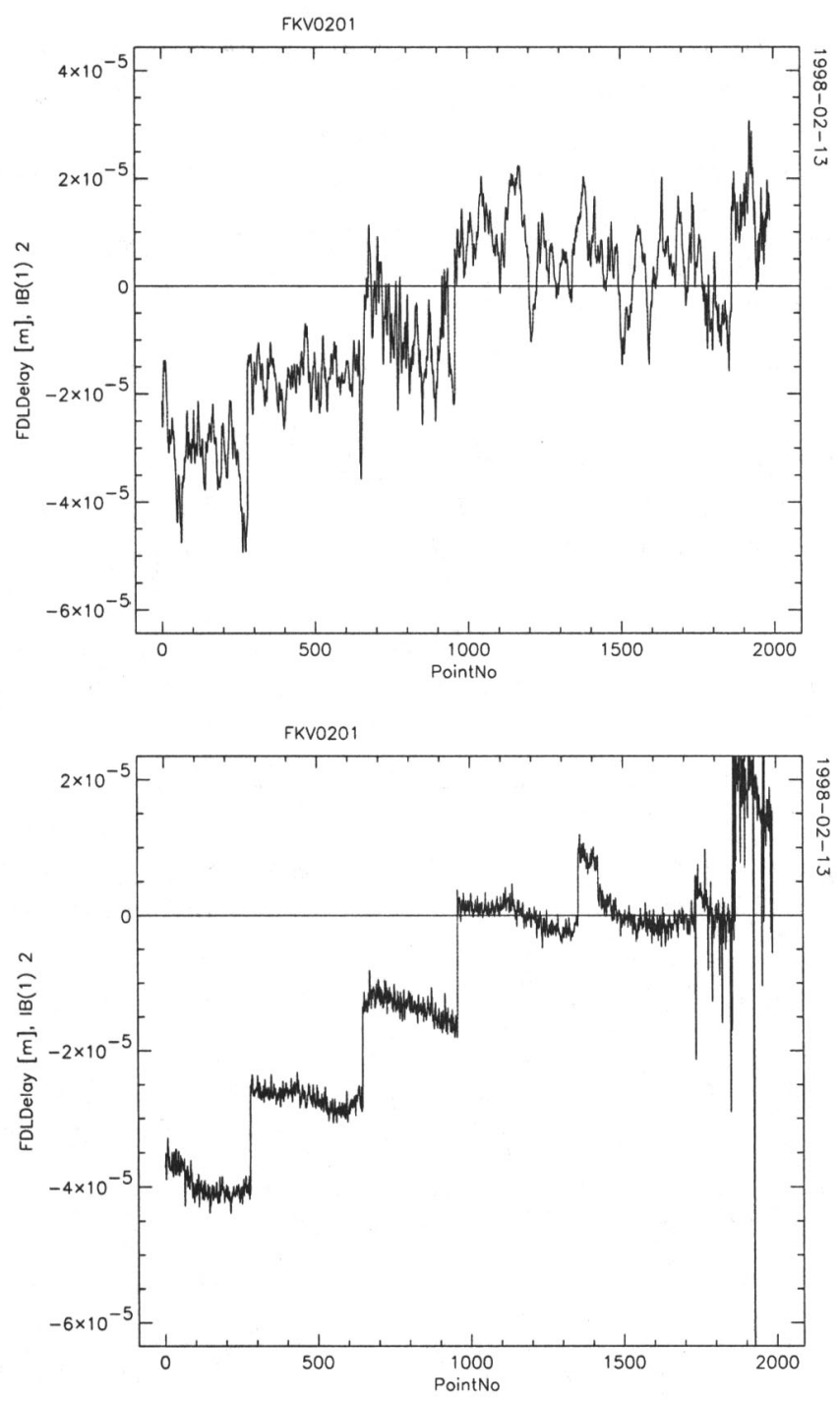

Figure 4. An example, for observations of FK5 201 on 02-13-98 UT, of the application of dispersion corrections to the observed delays (on the center-east baseline). Figure 4a (top) displays the residuals, relative to the calculated delay (estimated from the nominal star position, baseline, and time), for all of the $200 \mathrm{~ms}$ coherently averaged delays. (These data were collected during seven scans, spaced over four hours). Figure $4 \mathrm{~b}$ (bottom) displays the result of the application of the dispersion corrections to the uncorrected delays of Figure 4a. Note the much reduced, and more random, distribution of the points within each scan. (Discontinuities between scans are likely due to baseline motion, and uncertainties in the star's position.) 
tions (and resulting delay changes) can be reproduced to the level of $\sim 1 \mu \mathrm{m}$, even before dispersion compensation is applied to the measured delays.

Recent advances in all these techniques for the correction of stellar observations to the corresponding 'geometrical' delays promise the achievement of accurate wide-angle astrometric solutions in the very near future.

Acknowledgments. The NPOI project is funded by the Oceanographer of the Navy and the Office of Naval Research.

\section{References}

Armstrong, J. T., Mozurkewich, D., Rickard, L. J, Hutter, D. J., Benson, J. A., Bowers, P. F., Elias II, N. M., Hummel, C. A., Johnston, K. J., Buscher, D. F., Clark III, J. H., Ha, L., Ling, L.-C., White, N. M., \& Simon, R. S., 1998a, Astrophys. J., 496, 550.

Armstrong, J. T., Mozurkewich, D., Pauls, T. A., \& Hajian, A. R., 1998b, in Astronomical Interferometry, R. Reasenberg \& M. Shao, Bellingham: SPIE, 3350, 461.

Benson, J. A., Mozurkewich, D., \& Jefferies, S. M., 1998, in Astronomical Interferometry, R. Reasenberg \& M. Shao, Bellingham: SPIE, 3350, 493.

Clark III, J. H., Ha, L., Mozurkewich, D., \& Armstrong, J. T., 1998, in Astronomical Interferometry, R. Reasenberg \& M. Shao, Bellingham: SPIE, 3350, 497.

Colavita, M. M., Shao, M., \& Staelin, D. H., 1987, Appl. Opt., 26, 4113.

Danchi, W. C., Arthur, A., Fulton, R., Peck, M., Sadoulet, B., Sutton, E. C., Townes, C. H., \& Weitzmann, R. H., 1986, in Advanced Technology Optical Telescopes III, L. D. Barr, Bellingham: SPIE, 628, 422.

Elias II, N. M., 1994, in Amplitude and Intensity Spatial Interferometry II, J. B. Breckinridge, Bellingham: SPIE, 2200, 71.

Hines, B., Shao, M., \& Colavita, M. M., 1990, in Amplitude and Intensity Spatial Interferometry, J. B. Breckinridge, Bellingham: SPIE, 1237, 87.

Hummel, C. A., Mozurkewich, D., Elias II, N. M., Quirrenbach, A., Buscher, D. F., Armstrong, J. T., Johnston, K. J., Simon, R. S., \& Hutter, D. J., 1994, Astron. J., 108, 326.

Hutter, D. J., 1992, in High-Resolution Imaging by Interferometry II, J. M. Beckers \& F. Merkle, Garching: ESO, 39, 1187.

Mozurkewich, D., 1994, in Amplitude and Intensity Spatial Interferometry II, J. B. Breckinridge, Bellingham: SPIE, 2200, 76.

White, N. M., Mozurkewich, D., Armstrong, J. T., Hutter, D. J., Johnston, K. J., Clark III, J. H., \& Ha, L., 1998, in Astronomical Interferometry, R. Reasenberg \& M. Shao, Bellingham: SPIE, 3350, 471. 